\title{
O USO DE PINTURAS DE DALÍ COMO FERRAMENTA PARA AVALIAÇÃO DAS ALTERAÇÕES NA PERCEPÇÃO DE FORMA E TAMANHO EM PACIENTES ESQUIZOFRÊNICOS'
}

\author{
Maria Lúcia de Bustamante Simas \\ Renata M.T.B. L. Nogueira \\ Geórgia M.M.Menezes \\ Viviane F. Amaral \\ Aline M. Lacerda \\ Natanael A. Santos
}

Resumo: Estudos envolvendo portadores de esquizofrenia têm mostrado alterações severas da percepção e cognição. A maioria dos diagnósticos, entretanto, é baseada na avaliação de processos cognitivos. Nossos estudos investigaram a percepção alterada de forma e tamanho em pacientes com esquizofrenia e com depressão maior utilizando 24 pinturas de Salvador Dalí. Conduzimos dois estudos comparando grupos experimental e controle e observamos, em ambos os casos, que os portadores de esquizofrenia perceberam figuras 1,5 e 3,0 vezes maior do que os respectivos grupos controle. Em outro estudo com pacientes com depressão maior não foram observadas alterações na percepção de tamanho comparado ao 
grupo controle. Concluímos que este efeito de percepção de forma e tamanho alterados pode servir como marcador no diagnóstico precoce da presença de sintomas positivos na esquizofrenia. Sugerimos que estas pinturas de Salvador Dalí sejam utilizadas como ferramenta para avaliar alterações na percepção de formas e tamanhos em pacientes portadores de esquizofrenia e, assim, prevenir o agravamento dos sintomas cognitivos.

Palavras-chave: Esquizofrenia. Percepção. Forma. Tamanho. Pareidolia.

Estudos sobre a esquizofrenia revelam deficiências graves na percepção e cognição do paciente. O diagnóstico desta patologia é sistematicamente realizado com base em alterações cognitivas relacionadas ao discurso e ao pensamento do indivíduo. Neste estudo propomos uma nova forma de avaliação da esquizofrenia, considerando que as alterações cognitivas são necessariamente precedidas por modificações sensoriais auditivas e visuais. Sendo assim, por que não tratar o paciente com base apenas nestas alterações sensório-perceptuais incipientes? A dificuldade reside em detectar quais eventos seriam relevantes, ou não, no processo de agravamento dos sintomas positivos que frequentemente estão associados ao delírio, às ilusões e às alucinações. Nosso grupo vem investigando formas de se mensurar estas alterações de percepções visuais de forma e tamanho que precedem a eclosão do surto na esquizofrenia e que, por suas características, permitiriam o diagnóstico e o tratamento precoce do paciente.

O efeito que buscamos medir pode ser exemplificado na Figura 1, em que observamos o uso das bordas de vários objetos, em uma cena natural, para a organização perceptual de um único objeto, supostamente fictício, que assume proporções muito ampliadas. As pinturas de Salvador Dalí e Victor Molev ilustram com muita propriedade este efeito que chamaremos de assembling forms, em inglês, e concatenação de formas, em português. A Figura 2 mostra efeitos de concatenações de formas: as duas pinturas acima são de Salvador Dalí e as três abaixo de Victor Molev. 

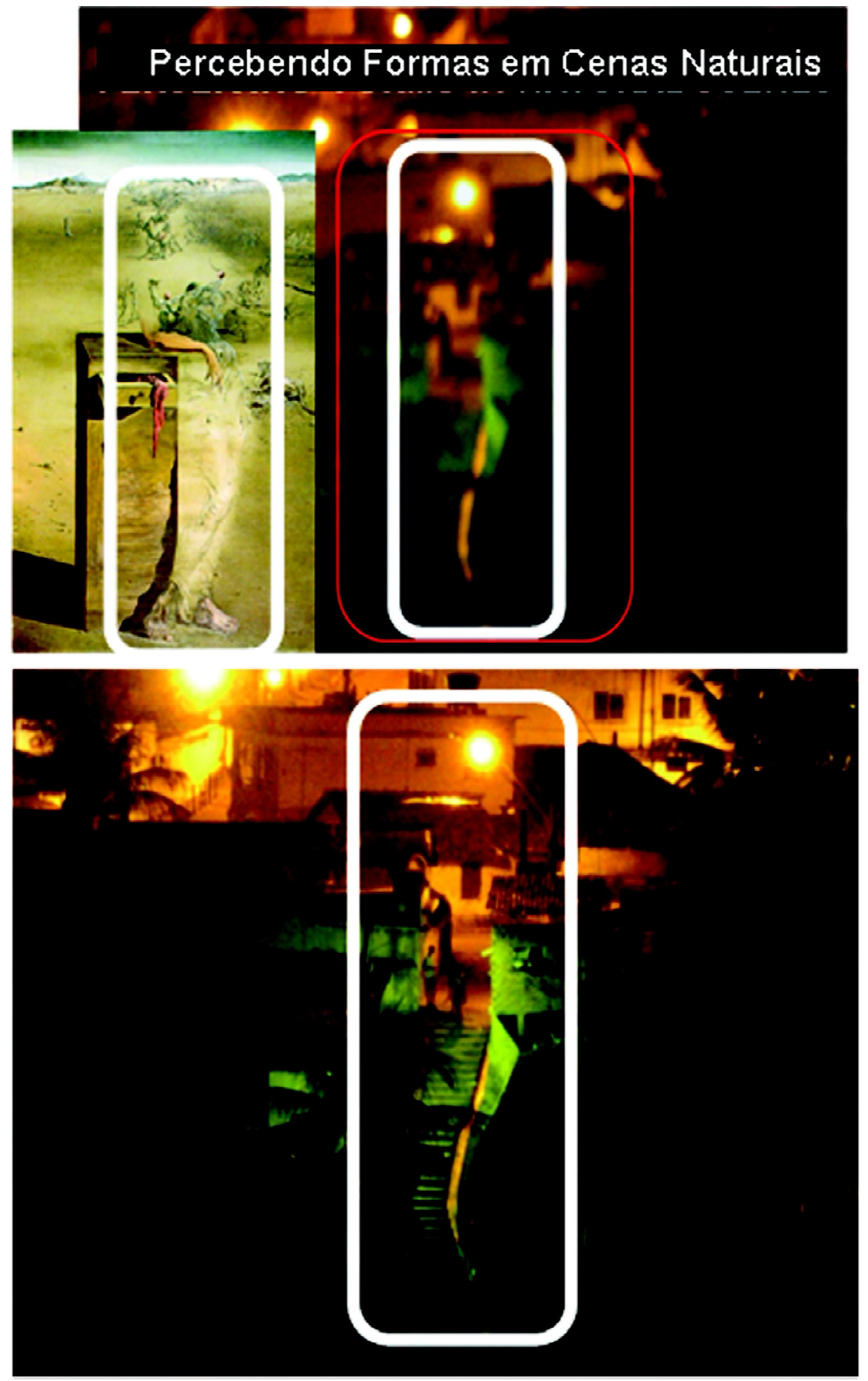

Figura 1. Exemplo de cena natural onde se formou a imagem semelhante à pintura "España" de Salvador Dalí. Observe a desproporção do tamanho da imagem com referência à escala da cena. 
A concatenação de formas na esquizofrenia resulta em objetos de tamanhos muito grandes. Ainda que se possa considerar como um efeito de dismetropsia, não se trata de macropsia porque não ocorre o aumento de um mesmo objeto e sim a percepção de um objeto diferente e muito aumentado em tamanho. Se considerarmos como "normal" um envelope de frequências espaciais com máximas sensibilidades em 3 ou 4 ciclos por grau de ângulo visual, cpg, nos casos da concatenação, os envelopes teriam máximos em 0,05 a 0,1 cpg, frequências não testadas na maioria dos estudos sobre deficiências da sensibilidade ao contraste na esquizofrenia.

Como aplicada à esquizofrenia, e em se tratando de percepção visual de forma e tamanho, preferimos chamar este fenômeno de concatenação de formas também para diferenciar daqueles já descritos na literatura como apofenia e pareidolia.

Apofenia se refere à atribuição, em qualquer modalidade perceptiva, de intencionalidade ou causalidade entre estímulos aleatórios. Fyfe, Williams, Mason e Pickup (2008) utilizaram este termo para estudar a vulnerabilidade à esquizofrenia avaliando a inabilidade de inferência sobre o estado mental do"outro" (Teoria da Mente [sic.]) e a tendência a atribuir significado a eventos não relacionados. Eles apresentaram dois conjuntos de tarefas, em um contexto cognitivo, em que o participante deveria fazer inferências de inter-relações entre objetos em movimento e classificá-las como sendo de natureza aleatória,física ou sob o rótulo"Teoria da Mente." A outra tarefa consistiu nestas mesmas classificações para responder perguntas sobre histórias (curtas). Eles concluíram que a ocorrência de apofenia era predita por aqueles voluntários que apresentavam alta esquizotipia e tendência ao delírio.

Pareidolia "é a percepção equivocada de algo claro e distinto a partir de um estímulo vago e obscuro" (Maranhão-Filho \& Vincent, 2009). Encontramos outra definição por Martín Araguz, Bustamante Martínez, Fernandéz-Armayor Ajo e López Gómez (2002), que caracterizam este fenômeno como a visualização involuntária e espontânea de uma percepção na qual a realidade e o sonho interagem. Estes autores também citam o fenômeno como fonte de inspiração de diferentes manifestações artísticas, assim como a base de algumas investigações psicológicas, como o teste de Rorcharch. As imagens da Figura 1 exigem concatenação e demonstram o efeito de tamanho de grande porte, e as pinturas apresentadas na Figura 2 são realmente manifestações artísticas mais elaboradas. Ambas não se enquadram perfeitamente nas definições de pareidolia. Além disso, o fenômeno que vamos mensurar tem como propriedade principal e intrínseca a percepção de tamanhos muito aumentados em relação ao contexto das figuras em sua vizinhança. 

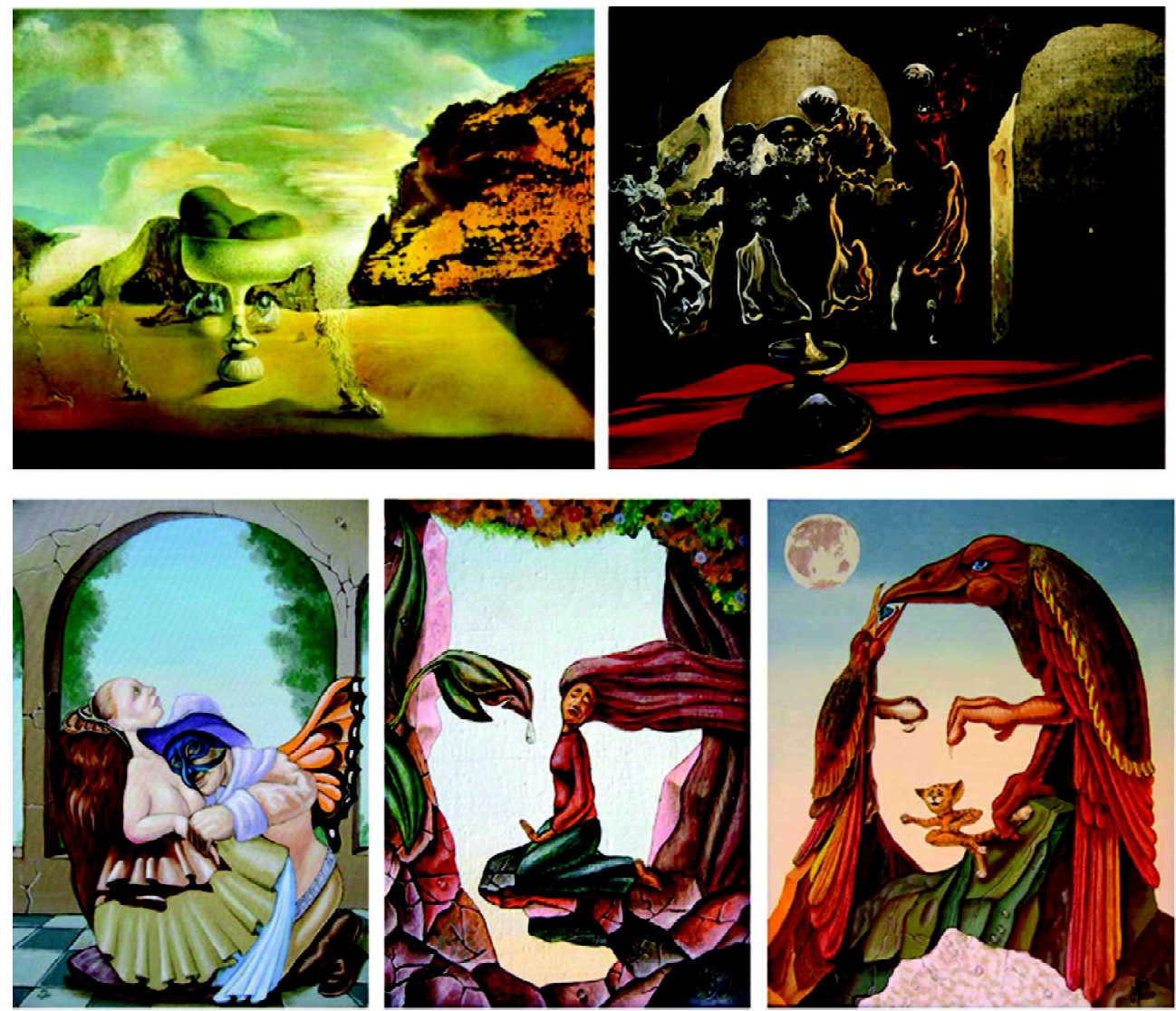

Figura 2. Exemplos de pinturas de Salvador Dalí (acima) e de Victor Molev (abaixo) onde ocorre a concatenação de formas e tamanho, com preferência por tamanhos de grande porte. Estes também seriam exemplos de pareidolia na visão.

Portanto, neste trabalho, para mensurar o efeito de concatenação de formas e dos tamanhos de grande porte das formas concatenadas, conduzimos três estudos, dois com portadores de esquizofrenia e um com portadores de depressão maior. Mensuramos o efeito com o uso de 24 pinturas de Salvador Dalí.

\section{Método}

Os Estudos 1 e 2 foram conduzidos com pacientes diagnosticados como portadores de esquizofrenia. Além disso, com o objetivo de investigar possíveis semelhanças ou diferenças sensório-perceptuais em pacientes com depressão maior, conduzimos também o Estudo 3, para efeito de comparação entre os três estudos. 


\section{Voluntários}

\section{Estudo 1}

Participaram deste estudo 60 voluntários com idades de 20 a 40 anos, sendo 30 pacientes (média $=31,23$ anos, $d p=6,78$ ), integrantes do grupo experimental, GE, diagnosticados pela CID-10 como portadores de esquizofrenia e frequentadores dos Centros de Atenção Psicossocial,CAPs, Recife, $\mathrm{PE}$, em regime de frequência de um ou dois turnos diurnos, com ensino fundamental incompleto. Os outros 30 participantes (média $=28,3$ anos, $\mathrm{dp}=5,35$ ) do grupo controle, $\mathrm{GC}$, não apresentavam desordens psiquiátricas e não utilizavam medicações relacionadas a patologias desta natureza, com escolaridade similar à do GE. As acuidades visuais eram normais ou corrigidas.

\section{Estudo 2}

Participaram deste estudo 44 voluntários com idades entre 30 e 60 anos, sendo 22 pacientes (média $=52,2$ anos, $d p=14,5$ ) portadores de esquizofrenia diagnosticada por médicos psiquiatras através da CID-10. Os voluntários do GE eram frequentadores do Ambulatório de Psiquiatria do Hospital das Clínicas da Universidade Federal de Pernambuco,UFPE, Recife, PE. Os outros 22 voluntários do GC (média $=50,10$ anos, dp $=12,7$ ) tinham escolaridade equivalente ao GE, isto é, $70 \%$ analfabetos, $28 \% \mathrm{com}$ ensino fundamental incompleto e $2 \%$ com ensino médio. As acuidades visuais eram normais ou corrigidas.

\section{Estudo 3}

Participaram deste estudo 40 voluntários adultos de ambos os sexos com idades entre 19 e 62 anos (média $=40,12$ anos, $d p=12,7$ ), 20 deles isentos de patologia (sem episódio depressivo - GC) e 20 com episódio depressivo, $\mathrm{GE}$, diagnosticados por médicos psiquiatras da cidade de João Pessoa de acordo com a CID-10. Além disso, os participantes de ambos os grupos responderam ao Inventário de Depressão de Beck (BDI) formado por 21 questões de múltipla escolha variando da letra "a" à "d"e retratando os sintomas mais acentuados da depressão. As acuidades visuais eram normais ou corrigidas. 


\section{Material e Estímulos}

\section{Estudo 1}

Foram utilizadas 24 pinturas de Salvador Dalí fotografadas e impressas na dimensão $10 \times 15 \mathrm{~cm}$. As pinturas foram selecionadas com base nas cenas que apresentavam a maior diversidade de figuras e tamanhos.

\section{Estudo 2}

As mesmas pinturas de Salvador Dalí do Estudo 1 foram utilizadas.

Além das fotografias, foi utilizada uma estante para partitura musical com o objetivo de apoiar as fotografias e pastas L Polibrás cristal (ref.: 0931), em polipropileno, $0,18 \mathrm{~mm}$, formato $220 \times 330 \mathrm{~mm}$, para se sobrepor a cada fotografia e proporcionar a marcação das respostas dos participantes com um marcador permanente.

\section{Estudo 3}

As mesmas pinturas de Salvador Dalí do Estudo 1 foram utilizadas, assim como uma estante de partitura, pastas $L$ e marcador permanente do Estudo 2.

\section{Procedimentos}

\section{Estudo 1}

As 24 fotografias das pinturas de Dalí foram apresentadas aos voluntários uma após a outra, sem limite de tempo para observação, a uma distância de 30 centímetros. Após a observação de cada fotografia o sujeito indicou a primeira figura que se destacou. A instrução fornecida aos participantes foi padronizada:" Você vai ver fotografias de pinturas de um pintor e depois de olhar cada uma você deverá indicar a primeira figura que mais chamou sua atenção". O tamanho registrado foi sempre o da primeira figura cuja observação foi relatada.

\section{Estudos 2 e 3}

Diferente do Estudo 1, as 24 fotografias das pinturas de Dalí foram apresentadas aos voluntários na estante de partitura, sucessivamente, sem limite de tempo para observação e resposta, a uma distância de $30 \mathrm{~cm}$ do olho do observador. As apresentações seguiram sempre a mesma ordem. O participante foi instruído a indicar a primeira figura que lhe chamou a 
atenção em cada pintura. A instrução fornecida aos participantes foi padronizada:"Você vai ver fotografias de quadros de um pintor e depois de olhar cada uma você deverá indicar a primeira figura, ou parte de uma figura, ou a primeira coisa que mais lhe chamou atenção". Após a indicação da figura pelo voluntário, o pesquisador a circulava com o marcador. E esse procedimento se repetiu em cada um dos 24 quadros. A Figura 3 ilustra o procedimento dos Estudos 2 e 3.

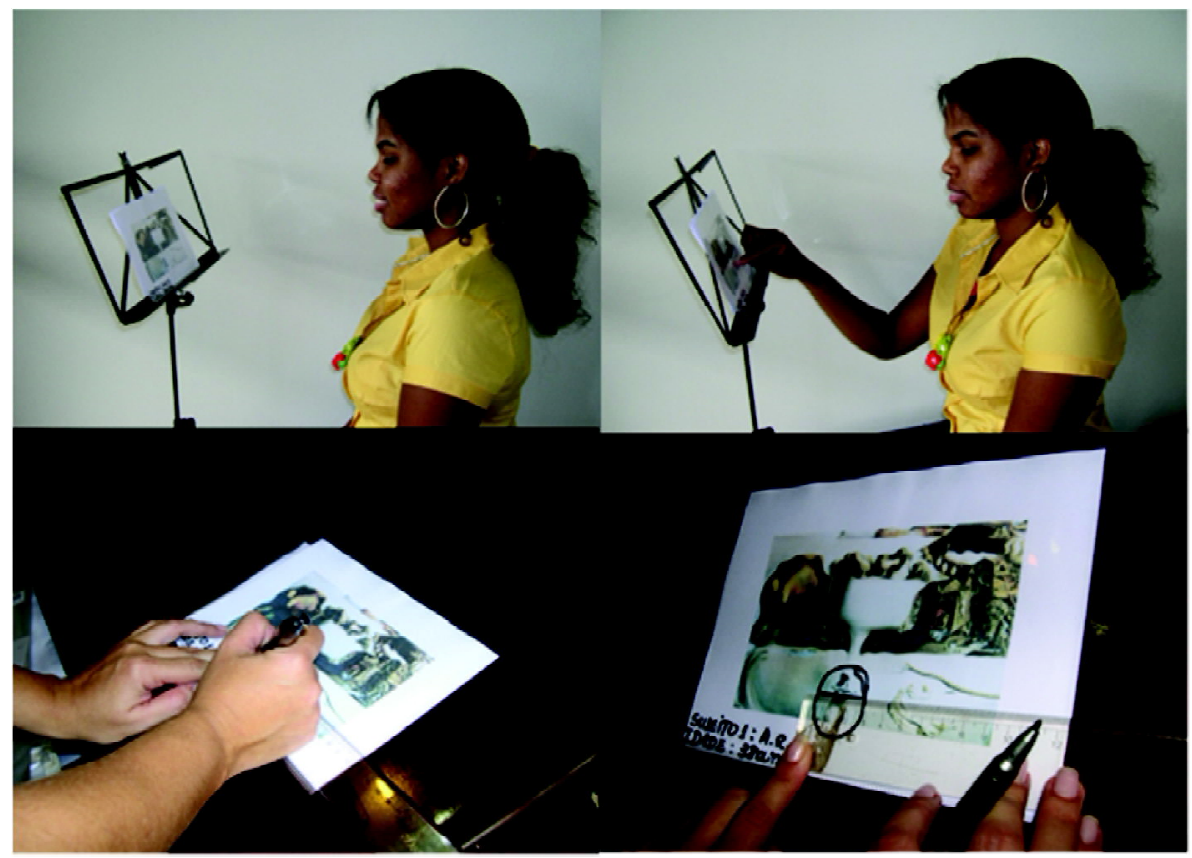

Figura 3. Fotos ilustrando o procedimento nos Estudos 2 e 3.

\section{Resultados}

Nos três estudos foram realizadas ANOVAS com dois fatores, um com repetição, os resultados mostraram diferenças significantes entre os grupos GC e GE $\left(F_{1,58}=550.62, \mathrm{p}<0.001\right),(\mathrm{F}(1,42)=548.17, \mathrm{p}<0.001)$ e $(\mathrm{F}(1$, $38)=4.28 ; p=0,04)$. Voluntários no $G E$ selecionaram tamanhos em média (10-23 $3^{\circ}$ graus de ângulo visual) cerca de três vezes maiores ( $F(23$, $1334)=14.864, p<0.001)$ do que aqueles selecionados pelo GC $\left(4-5^{\circ}\right.$ graus de ângulo visual), enquanto, no Estudo 2 , as formas selecionadas foram 1.5 maiores do que o $C G(F(23,966)=12.23, p<0.001)$. Não houve diferenças significantes na interação tamanho $x$ grupo no estudo com portadores de depressão maior, $(F(23,874)=1.54, p>0.05)$. Comparando os resultados dos Estudos 2 e 3, ajustando para igualar o $\mathrm{N}$ dos grupos, pacientes portadores de esquizofrenia perceberam formas maiores do que os pa- 
cientes com depressão maior, $(F(23,966)=4.0, p<0.001)$, mas não houve diferença significante entre os respectivos $G C,(F(1,42)=0.12, p=0.73)$.

A Figura 4 mostra os tamanhos selecionados em cada um dos três estudos convertidos em frequências espaciais fundamentais através da operação 1/tamanho selecionado em graus de ângulo visual. Os gráficos destacam os valores de frequências espaciais iguais ou menores do que $0,1 \mathrm{cpg}$. No Estudo 1, apenas 2 tamanhos da figura escolhida em primeiro lugar estão acima da frequência espacial fundamental de 0,1 cpg. No Estudo 2, apenas 1 tamanho é maior que a frequência espacial fundamental de 0,1 cpg. No Estudo 3, de pacientes com depressão maior, 9 tamanhos das figuras escolhidas em primeiro lugar correspondem a frequências espaciais fundamentais maiores que $0,1 \mathrm{cpg}$. Por outro lado, apesar da maior variabilidade dos tamanhos escolhidos pelo GC no Estudo 3, não houve diferenças significantes entre os GC deste estudo e do Estudo 2 quando o $\mathrm{N}$ foi ajustado para permitir comparações em uma ANOVA. 

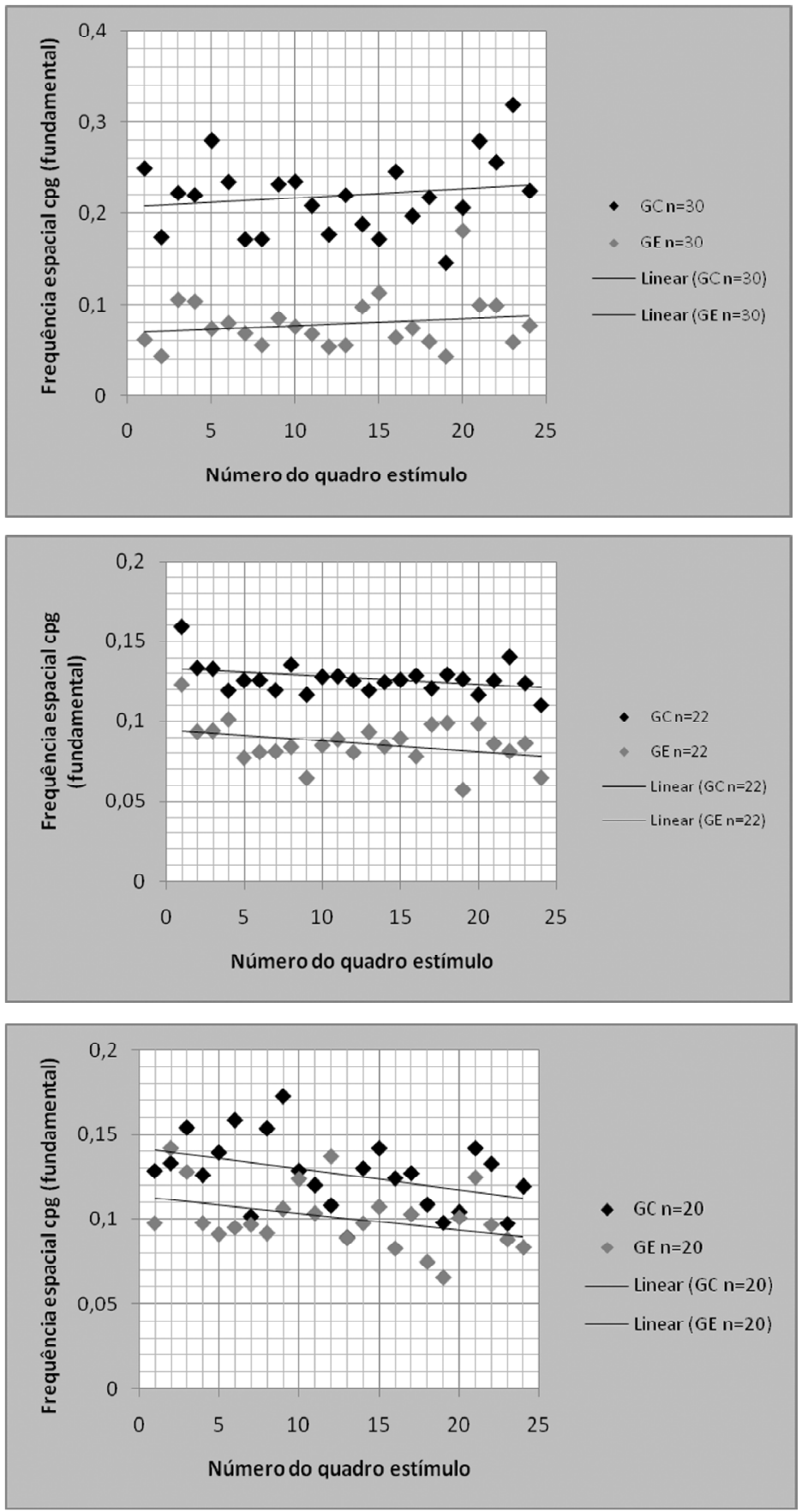

Figura 4. Gráficos ilustrando as faixas de frequências espaciais fundamentais das primeiras figuras apontadas pelos voluntários nos GC e GE nos três estudos. Estudo 1 (acima), Estudo 2 (centro), Estudo 3 (abaixo). As áreas com fundos cinza realçam os valores de frequências espaciais fundamentais abaixo de $0,1 \mathrm{cpg}$. 


\section{Discussão}

Existem três premissas centrais aos estudos aqui relatados: (1) a de que as deficiências perceptivas na esquizofrenia ocorrem necessariamente antes dos déficits cognitivos; (2) a de que durante o agravamento dos sintomas positivos da esquizofrenia (isto é, delírios, alucinações e ilusões), ocorre um fenômeno semelhante à pareidolia que, aplicado à visão, denominamos concatenação de formas; e, finalmente, (3) a de que existe uma percepção espontânea de figuras com tamanhos de grande porte e que, provavelmente, esta alteração perceptiva sinaliza um deslocamento do envelope de frequências espaciais no sentido de maior sensibilidade ao contraste para as faixas de frequências espaciais baixíssimas, supostamente com sensibilidades máximas na faixa do espectro inferior a 0,1 cpg. Por isso, nossos estudos se centraram na mensuração do tamanho das primeiras figuras observadas. Nas 24 pinturas de Salvador Dalí que utilizamos, existem efeitos que poderiam se enquadrar como pareidolia, e que exigem a ocorrência de concatenação de formas, mas também existe uma grande variedade de figuras e tamanhos que não são, necessariamente, exemplos de pareidolia, mas, ainda assim, induzem na esquizofrenia a percepção dos tamanhos maiores.

Os resultados de nossos estudos mostram uma seletividade do efeito de concatenação de formase da percepção alterada para tamanho de grande porte ocorrendo nos Estudos 1 e 2 com portadores de esquizofrenia. Isso não ocorreu com os pacientes de depressão maior. Foi possível demonstrar a diferença significativa entre os pacientes com esquizofrenia do Estudo 2 e daqueles com depressão maior no Estudo 3 na seleção das primeiras figuras percebidas. Também foi possível demonstrar que não houve diferença significativa entre os tamanhos das figuras escolhidas pelos GC dos Estudos 2 e 3. Não comparamos ao Estudo 1 devido à diferença de procedimentos.

Nossos resultados favorecem a possibilidade de se utilizar esses estímulos e procedimentos como marcadores da presença de sintomas positivos na esquizofrenia e como subsídio no diagnóstico e na tomada de decisões quanto ao tratamento inicial ou de manutenção assim como à medicação a ser adotada ou modificada para cada paciente.

Finalmente, cabe mencionar que um trabalho recente de Koychev, El-Deredy, Haenschel e Deakin (2010), com potenciais visuais provocados, encontra como resultados da estimulação de pacientes portadores de esquizotipia evidência de que essa patologia seria caracterizada por/ pelas alterações sensoriais que se refletem na amplitude reduzida de P1, mas na normalidade dos componentes N1 e P2. Esses autores sugerem, como em nossa premissa, que a desorganização cognitiva resultaria dos déficits sensoriais de latências muito breves (e.g.até $150 \mathrm{~ms}$ ). 
Dali paintings as a tool for assessing altered form and size perception in schizophrenic patients

Abstract: A variety of studies involving schizophrenic patients have shown the occurrence of severe disabilities related to perception and cognition. Most diagnostics rely on alterations that reveal faulty reasoning. Our studies evaluate altered visual form and size perception in schizophrenic and major depression patients using 24 paintings by Salvador Dalí. We conducted two studies comparing experimental and control groups and found in both cases that schizophrenic patients first saw forms 1.5-3.0 times larger than controls. In another study with major depression patients, we show the absence of alterations in size perception during aggravated depressive symptoms. These effects on size perception can contribute as markers in the precocious diagnostic of the onset of positive symptoms in schizophrenia. We suggest that these paintings by Dalí can be employed as a tool to assess the state of visual form and size perception in schizophrenic patients, and therefore prevent cognitive deterioration by introducing early treatment.

Keywords: Schizophrenia. Perception. Shape. Size. Pareidolia.

\section{L'Utilizacion des paintures de Dalí pour avaliacion de la percepcion de forme et grandeur dans la esquizofrenie}

Résumé: Les études de patients schizophrènes ont montré d'importantes modifications dans la perception et la cognition. La plupart des diagnostics, toutefois, est basé sur l'évaluation des processus cognitifs. Nous avons utilisé 24 tableaux de Salvador Dalí pour étudier les modifications en la perception de forme et dimension des patients schizophrènes et de patients souffrant de dépression majeure. Ici sont présentés deux études comparant des groupes expérimental et de contrôle. Dans les deux, patients schizophrènes ont vu les images présentées comme étant de 1,5 et 3,0 fois plus grand par rapport au groupe contrôle. Dans une autre étude, les patients souffrant de dépression majeure n'ont montré aucune changement en dimension perçue en comparaison au groupe de contrôle. Nous concluons que les changements dans la perception de forme et dimension peuvent servir de marqueur pour le diagnostic précoce des symptômes positifs de la schizophrénie. Nous suggérons que ces peintures de Salvador Dalí sont utilisés comme un instrument pour évaluer les changements dans la perception de formes et de dimensions des patients schizophrènes et d'éviter ainsi l'aggravation des symptômes cognitifs.

Mots-clés: Schizophrénie. Perception. Forme. Taille. Pareidolia. 
El uso de las pinturas de Dalí como un instrumento para evaluar los cambios en la percepción de la forma y el tamaño en los pacientes esquizofrénicos

Resumen: Estudios con pacientes con esquizofrenia han mostrado cambios severos en la percepción y la cognición. La mayoría de los diagnósticos, sin embargo, se basa en la evaluación de los procesos cognitivos. Hemos utilizado 24 pinturas de Salvador Dalí para investigar los cambios en la percepción de la forma y el tamaño en los pacientes esquizofrénicos y con depresión mayor. Aquí se presentan dos estudios que compararon grupos experimental y control. En ambos, observamos que los pacientes con esquizofrenia vieron las imágenes presentadas como 1.5 y 3.0 veces mayores en comparación con los grupos control. En otro estudio, los pacientes con depresión mayor no mostraron cambios en el tamaño percibido en comparación con el grupo control. Se concluye que los cambios en la percepción de la forma y el tamaño pueden servir como un marcador para el diagnóstico precoz de los síntomas positivos en la esquizofrenia. Sugerimos que estas pinturas de Salvador Dalí sean utilizadas como un instrumento para evaluar los cambios en la percepción de formas y tamaños en los pacientes esquizofrénicos y así prevenir el agravamiento de los síntomas cognitivos.

Palabras clave: Esquizofrenia. Percepción. Forma. Tamaño. Pareidolia.

\section{Referências}

Fyfe, S., Williams, C., Mason, O.J., \& Pickup, G.J. (2008). Apophenia, theory of mind and schizotypy:Perceiving meaning and intentionality in randomness.Cortex, 44, 1316-1325.

Koychev, I., El-Deredy, W., Haenschel, C., \& Deakin, J.F.W.(2010).Visual information processing deficits as biomarkers of vulnerability to schizophrenia:An eventrelated potential study in schizotypy. Neuropsychologia, 48,2205-2214.

Maranhão-Filho, P., \& Vincent, M. B. (2009). Neuropareidolia. Arquivos de Neuropsiquiatria, 67(4), 1117-1123.

Martín Araguz, A., Bustamante Martínez,M.C., Fernandéz-Armayor Ajo, V., \& López Gómez, M. (2002). Pareidolia in Beato de Liébana's illuminated Visigothic codes. Neurologia, 17(10),633-42. 
Maria Lúcia de Bustamante Simas, Laboratório de Percepção Visual, LabVis-UFPE, Departamento de Psicologia, Universidade Federal de Pernambuco. Endereço para correspondência: Rua Gomes de Matos Jr, 91/502, Rosarinho, Recife, CEP: 52050-4 PE, Brasil.Endereço eletrônico: maria.simas@pq.cnpq.br

Renata M.T. B. L. Nogueira, Laboratório de Percepção Visual, LabVis-UFPE, Departamento de Psicologia, Universidade Federal de Pernambuco. Endereço para correspondência: LabVis-UFPE, Rua Professor Moraes Rego, s/n, Departamento de Psicologia, $\mathrm{CFCH} 9^{\circ}$ andar, Cidade Universitária, Universidade Federal de Pernambuco, Recife, 50670 901,PE, Brasil. Endereço eletrônico: rm_toscano@yahoo.com.br

Geórgia M.M. Menezes, Laboratório de Percepção Visual, LabVis-UFPE, Departamento de Psicologia, Universidade Federal de Pernambuco. Endereço para correspondência: LabVis-UFPE, Rua Professor Moraes Rego, s/n, Departamento de Psicologia, CFCH $9^{\circ}$ andar, Cidade Universitária, Universidade Federal de Pernambuco, Recife, 50670 901,PE, Brasil. Endereço eletrônico: geosenna@bol.com.br

Viviane F. Amaral, Laboratório de Percepção Visual, LabVis-UFPE, Departamento de Psicologia, Universidade Federal de Pernambuco. Endereço para correspondência: LabVis-UFPE, Rua Professor Moraes Rego, s/n, Departamento de Psicologia, CFCH $9 \circ$ andar, Cidade Universitária, Universidade Federal de Pernambuco, Recife, 50670 901, PE, Brasil. Endereço eletrônico: vivi_kiks@hotmail.com

Aline M. Lacerda, Departamento de Psicologia, Universidade Federal da Paraíba. Endereço para correspondência: Universidade Federal da Paraíba - Campus I, Rua Jardim Universitário, s/n, bairro Castelo Branco, João Pessoa/PB, Brasil, 58051-900.

Natanael A. Santos, Departamento de Psicologia, Universidade Federal da Paraíba. Endereço para correspondência: Universidade Federal da Paraíba - Campus I, Rua Jardim Universitário, s/n, bairro Castelo Branco, João Pessoa/PB, Brasil, 58051-900. Endereço eletrônico: natanael.santos@uol.com.br 